\title{
Síndrome polidípsico-poliúrico en trastornos de la conducta alimentaria de la adolescencia
}

\section{Polyuropolidipsic syndrome in adolescence eating in disorders}

\author{
Sandoval $L,{ }^{1}$ Calderón $A,{ }^{2}$ Argüello $L,{ }^{3}$ Soler $A,{ }^{4}$ Forero $C,{ }^{5}$ Céspedes $C^{6}$
}

\author{
${ }^{1}$ Residente de Pediatría, Pontificia Universidad Javeriana; Bogotá, \\ Colombia. \\ ${ }^{2}$ Residente de Pediatría, Pontificia Universidad Javeriana; Bogotá, \\ Colombia. \\ ${ }^{3}$ Residente de Pediatría, Pontificia Universidad Javeriana; Bogotá, \\ Colombia. \\ ${ }^{4}$ Médica pediatra, Hospital Universitario San Ignacio. Profesor \\ instructor, Pontificia Universidad Javeriana; Bogotá. Colombia. \\ ${ }^{5}$ Endocrinóloga pediatra, Hospital Universitario San Ignacio. \\ Profesor de la Facultad de Medicina, Pontificia Universidad \\ Javeriana; Bogotá. Colombia. \\ ${ }^{6}$ Endocrinóloga pediatra, Hospital Universitario San Ignacio. \\ Profesor de la Facultad de Medicina, Pontificia Universidad \\ Javeriana; Bogotá. Colombia. \\ Autor de correspondencia: Liliana Sandoval, MD \\ Correo electrónico: liliana.sandoval@javeriana.edu.co \\ Fecha de recepción: 12/01/2020 \\ Fecha de aceptación: 25/02/2020
}

\section{Resumen}

Los trastornos de la conducta alimentaria son un espectro de enfermedades psiquiátricas poco frecuentes, con una prevalencia difícil de establecer en la población pediátrica. Estos desórdenes pueden tener diversas repercusiones sistémicas, dentro de las que se encuentran las alteraciones endocrinológicas. Se presenta el caso de una adolescente remitida a urgencias desde la consulta externa de endocrinología por polidipsia y poliuria. Durante la hospitalización se descartan diabetes insípida, diabetes mellitus, síndrome de secreción inadecuada de la hormona antidiurética y cerebro perdedor de sal. Por tanto, una vez excluidas las causas orgánicas, se identifica un trastorno de la conducta alimentaria que podría cursar con polidipsia psicógena.

Palabras clave: trastornos de alimentación y de la ingestión de alimentos, trastornos de ingestión y alimentación en la niñez, polidipsia psicogénica, polidipsia, hiponatremia.

\section{Abstract}

Eating disorders are a spectrum of infrequent psychiatric diseases, with a prevalence that is difficult to establish in the pediatric population. They may have various systemic manifestations. We present a case of a teenager female who was referred from ambulatory endocrinology consult to the emergency department because of polydipsia and polyuria. During hospitalization, central and nephrogenic diabetes insipidus, diabetes mellitus, inappropriate antidiuretic hormone secretion syndrome and cerebral salt wasting syndrome were ruled out. Once the organic causes were excluded, an eating disorder with psychogenic polydipsia was identified.

Keywords: feeding and eating disorders, feeding and eating disorders of childhood, polydipsia, psychogenic, polydipsia, hyponatremia.

\section{Introducción}

Los trastornos de la conducta alimentaria (TCA) son un espectro de enfermedades de origen multifactorial en el que intervienen factores emocionales, psicosociales y conductuales (1). Se caracterizan por una alteración en la ingesta de alimentos, por lo que ocurre una disminución de sustratos energéticos. Esto conduce a cambios adaptativos en diferentes sistemas con el fin de conservar las funciones vitales. En la población pediátrica, la prevalencia de los TCA es difícil de establecer, sin embargo, son más frecuentes en el sexo femenino (2). La importancia de la detección temprana se asocia a un mejor pronóstico y al restablecimiento de la homeostasis corporal (3).

\section{Presentación del caso}

Se presenta una paciente de 14 años con un cuadro clínico de 4 meses de una pérdida de peso que alcanza los $12 \mathrm{~kg}$. La joven asiste a medicina general y allí se solicita la prueba de la hormona estimulante de la tiroides (TSH) que estaba en el 
límite inferior. Por tal motivo, se amplían los estudios de $\mathrm{T}_{4}$ total, $\mathrm{T}_{4}$ libre, $\mathrm{T}_{3}$ libre, tiroglobulina y anticuerpos tiroideos antiperoxidasa, con los cuales se realiza el diagnóstico de hipertiroidismo y se inicia el manejo con metimazol.

La paciente es valorada de forma extrainstitucional por endocrinología pediátrica y allí se descarta la existencia de hipertiroidismo, pues se observan niveles de hormonas tiroideas normales, razón por la cual se suspende el manejo. En esa oportunidad, la adolescente refiere 3 meses de amenorrea, 1 mes de hipergeusia, 2 semanas de polidipsia de predominio diurno y poliuria. En consecuencia, es remitida a urgencias para descartar una diabetes insípida.

Durante la estancia hospitalaria, se documenta la existencia de una osmolaridad sérica baja y niveles de sodio sérico limítrofes bajos y una glucemia normal (83-79 mg/dL), por lo que se descarta la diabetes mellitus. La paciente es evaluada por múltiples subespecialidades, incluyendo endocrinología pediátrica, dada la persistencia de poliuria, y se realiza la prueba de privación hídrica en la que se evidencia hipotensión, sed intolerable y taquicardia a las 2 horas de inicio, por lo que se suspende la fase de sed y se comienza la reposición para permitir la ingesta de 1,5 veces del volumen excretado.

Se realizan los paraclínicos de las 6 y 8 horas luego del inicio de la prueba, y se observan hiponatremia severa y osmolaridad sérica baja, registrando glucemias normales durante la totalidad de la prueba (Figuras 1-3). Después de ello, se suspende la prueba de privación hídrica, sin llegar a la fase de administración de desmopresina, y se descarta una diabetes insípida.

En 24 horas se registra una ingesta de 12,4 L, con un gasto urinario de 13,2 $\mathrm{mL} / \mathrm{kg} / \mathrm{h}$. Entre tanto, nefrología pediátrica considera la presencia de hiponatremia hipotónica con osmolaridad urinaria y sodio en orina bajo. Se indica la restricción hídrica con la corrección de la alteración hidroelectrolítica y se observa un gasto urinario normal, razón por la cual se descarta una secreción inadecuada de la hormona antidiurética (SIADH), así como cerebro perdedor de sal. Además, se realiza una resonancia magnética cerebral y de silla turca que reporta una leucoencefalopatía de probable origen microangiopático, de sustancia blanca del lóbulo frontal izquierdo, por ello se solicitan estudios complementarios que descartan una enfermedad autoinmune.

La paciente es valorada en psiquiatría infantil que considera realizar una reacción de ajuste. Se reinterroga tanto a ella como a sus familiares, quienes confirman que 8 meses antes de su ingreso hospitalario inició una dieta vegana. Al presentarse una pérdida significativa de peso, luego de 4 meses, la madre retomó la alimentación habitual, pero presentó hiporexia, dieta fraccionada, hipergeusia, pérdida del cabello, potomanía (durante 1 mes, aproximadamente $12 \mathrm{~L}$ diarios) y poliuria secundaria. La paciente refiere que se encontraba muy delgada y su deseo era aumentar de peso. Se consideró entonces un TCA con polidipsia psicógena, por lo cual se solicita una nueva valo-

Figura 1. Niveles de osmolaridad sérica durante la prueba de restricción hídrica hasta la octava hora: por osmolaridades persistentemente bajas, se suspende sin llegar a la fase de administración de desmopresina.

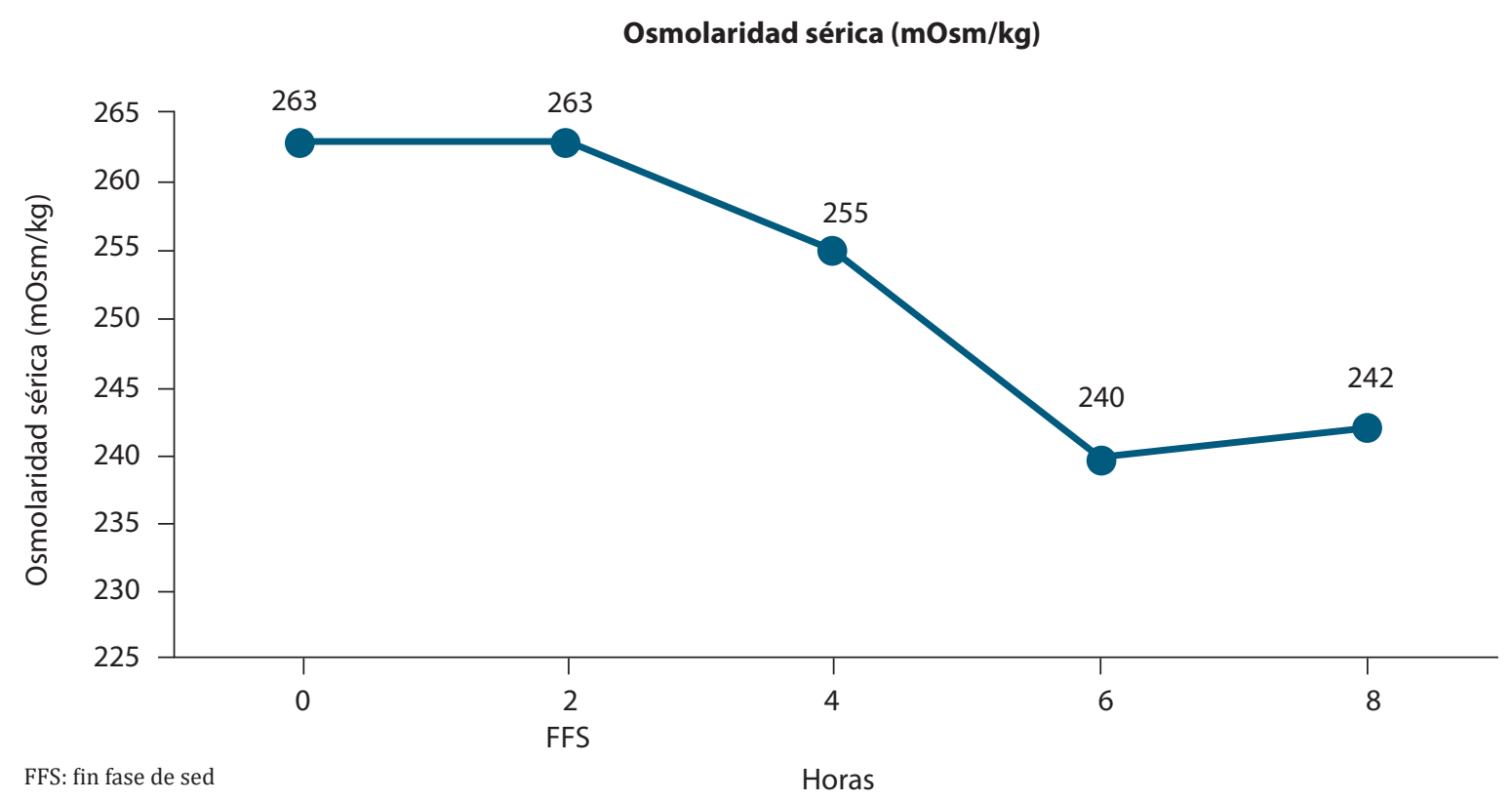


Figura 2. Niveles de sodio sérico durante la prueba de restricción hídrica hasta la octava hora: por niveles persistentemente bajos, se suspende sin llegar a la fase de administración de desmopresina.

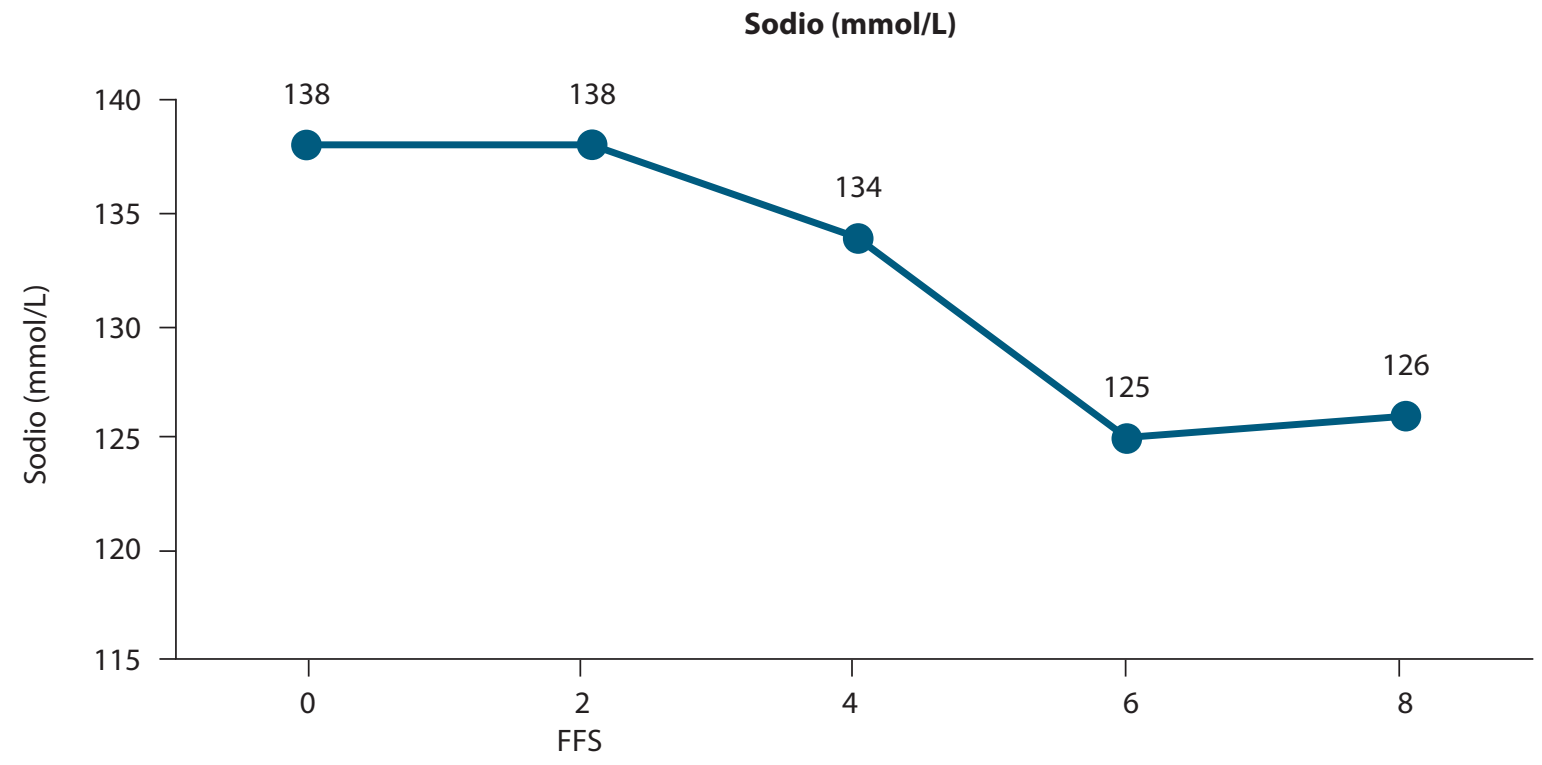

FFS: fin fase de sed

Horas

Figura 3. Niveles de glucemia durante la prueba de restricción hídrica hasta la octava hora.

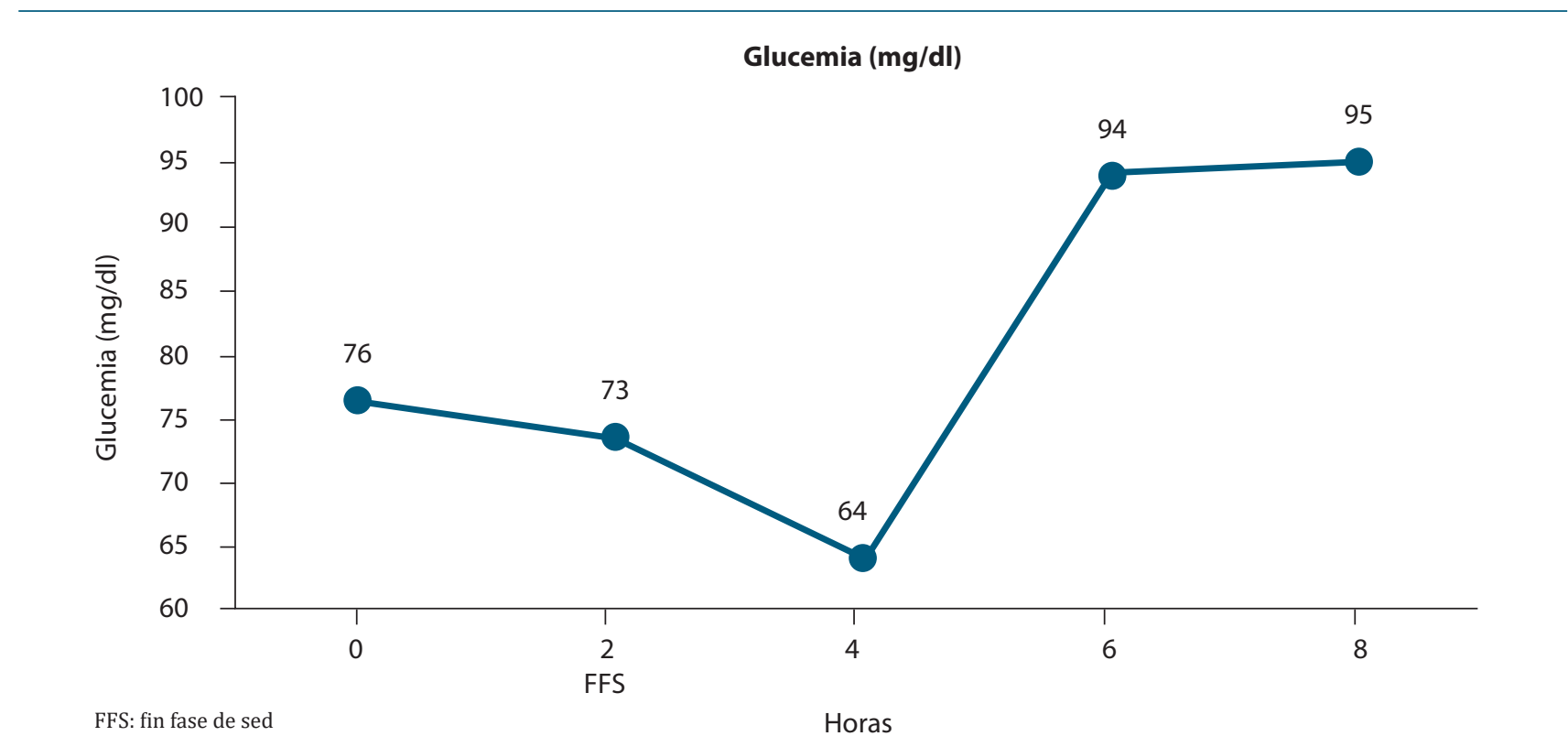

ración de psiquiatría infantil que encuentra síntomas sugestivos de trastorno depresivo del adolescente e inicia manejo con sertralina y psicoterapia conductual. La paciente presenta una disminución en la ingesta de líquidos y un aumento en el con- sumo de alimentos logrando una ganancia de peso del 1,6 \% respecto al inicial y se da egreso con un manejo farmacológico y psicoterapia personal y familiar. 


\section{Discusión}

Los TCA se caracterizan por la alteración en la ingesta de alimentos, lo que afecta la salud y el funcionamiento psicosocial (1). Su prevalencia en niños y adolescentes es difícil de establecer, dado el gran subdiagnóstico. Dentro de estos desórdenes se identifican la anorexia nerviosa (AN), la bulimia nerviosa y los trastornos de alimentación no especificados (TANE) $(2,4)$. Este grupo de enfermedades tiene manifestaciones multisistémicas. Entre ellas se encuentran las endocrinológicas, las cuales han sido estudiadas principalmente en relación con la AN, mientras que su severidad está asociada con el grado de caquexia del paciente (3). En el eje hormona de crecimiento $(\mathrm{GH})$ - factor de crecimiento similar a la insulina 1 (IGF-1) se presenta una resistencia periférica de la acción de la $\mathrm{GH}$, a pesar de tener niveles normales o elevados de esta, con una disminución de los niveles de IGF-1, por la baja expresión de los receptores hepáticos de la GH (4-6).

Durante la inanición, el organismo se compensa disminuyendo la producción de estrógenos, dada la supresión del eje hipotálamo-hipófisis-ovario (EHHO), lo que se asocia al hipogonadismo hipogonadotropo. Esto ocasiona amenorrea primaria, secundaria y disfunción menstrual, la cual se encuentra no solo en la AN sino en los demás TCA como lo presentó nuestra paciente $(3,5,7)$. En la población pediátrica se producirá un retraso o una regresión puberal por una disminución de los niveles de testosterona y estrógenos (4).

Asimismo, en el eje hipotálamo-hipofisario-adrenal se halla un aumento en las concentraciones de cortisol, las cuales son inversamente proporcionales a los niveles de glucemia en ayunas. Este se constituye en un mecanismo adaptativo para mantener la euglucemia (4). En cuanto al eje hipotálamo-hipófisis-tiroideo, en el caso descrito se reportaron niveles de TSH en el límite inferior y T3 y T4L normales, lo que es acorde con lo expresado en la literatura en TCA tipo AN, en la cual, existe un patrón eutiroideo enfermo secundario a la inanición crónica como mecanismo de adaptación a un estado de baja energía que ayuda a conservarla para funciones vitales $(6,7)$.

El síndrome polidípsico-poliúrico (SPP) se define como un aumento del volumen de la diuresis ( $>30-50 \mathrm{~mL} / \mathrm{kg} / \mathrm{d})$ no concentrada, acompañado de un incremento en la ingesta de líquidos. Esta puede ser la manifestación de diabetes insípida central por secreción insuficiente de la hormona antidiurética $(\mathrm{ADH})$, de diabetes insípida nefrogénica secundaria a la insensibilidad de la ADH a nivel renal y de polidipsia primaria o psicogénica, descrita en pacientes con trastornos psiquiátricos, entre ellos los de TCA.

Para establecer el origen del síndrome, en un principio se deben descartar otras causas relacionadas con la secreción y la acción de la $\mathrm{ADH}$, como la hiperglucemia o la hiperpotasemia. Luego de ello, se realiza una prueba de privación hídrica $(8,9)$, la cual se hizo a la paciente descartando diabetes insípida, esta es considerada el patrón de referencia para el diagnóstico diferencial del SPP y demuestra de forma indirecta la actividad de la ADH a través de la medición de la osmolaridad urinaria y, de esta forma, evalúa la capacidad de concentración en períodos de deshidratación (5). En el caso de la diabetes insípida central, la osmolaridad urinaria no sobrepasa la osmolaridad sérica durante la fase de deshidratación, pero aumenta en un 50 \% luego de la administración de la desmopresina. En la diabetes insípida nefrogénica, la osmolaridad urinaria será menor que la sérica, con un aumento $<50 \%$ después del manejo con desmopresina. En la polidipsia primaria o psicogénica se encuentra una osmolaridad urinaria mayor a la sérica, con un incremento $<10 \%$ luego de la administración de desmopresina (6).

La polidipsia primaria o psicogénica se caracteriza por el consumo excesivo de líquidos y poliuria secundaria durante un tiempo prolongado y en ausencia de una causa orgánica (5). Se reporta una incidencia de entre el 6-20 \% en pacientes psiquiátricos, entre los que se hallan aquellos pacientes con $\mathrm{AN}$ y esquizofrenia $(3,10-16)$. Dentro de la patogénesis, se ha descrito una desregulación del eje hipotálamo-hipófisis, sed y un umbral de sed más bajo (3). La polidipsia puede conducir a una disminución de la ADH y resultar en poliuria hipoosmolar (7). Al contrario de la diabetes insípida central, en la polidipsia psicogénica la poliuria es lenta y no se presenta nicturia ni ingesta de líquidos en las noches, así como tampoco fiebre $(10,11)$.

La complicación más frecuente de la polidipsia psicogénica es la hiponatremia hipotónica leve, la cual se asocia a una mayor tasa de rehospitalización y a un aumento de la morbilidad. Sin embargo, la de mayor mortalidad es la hiponatremia severa secundaria a intoxicación por agua, que se caracteriza clínicamente por confusión, letargia, psicosis, convulsiones, edema cerebral y, de forma menos frecuente, rabdomiólisis (3, 10, 12-16). Durante la hospitalización, la paciente cursó con hiponatremia hipoosmolar leve, como parte de su SPP, sin llegar a presentar signos ni síntomas de intoxicación por agua.

El tratamiento de la polidipsia primaria o psicogénica tiene resultados variables y consiste en el uso antipsicóticos atípicos para manejar la ansiedad. Además, se requiere de una terapia comportamental concomitante con psicoeducación del paciente y de la familia, la cual fue implementada para el caso descrito (17).

Dentro de lo revisado en la literatura colombiana y latinoamericana no se encontraron reportes de polidipsia primaria o psicogénica asociada a TCA; solo relacionada a esquizofrenia y trastorno esquizoafectivo $(12,15,16)$. Hay que tener en cuenta que al presentar esta entidad es importante descartar patologías psiquiátricas, dado su relación con las mismas para prevenir complicaciones multisistémicas, algunas de las cuales, pueden llevar a desenlaces fatales.

\section{Conflicto de intereses}

Los autores declaran que no tienen conflictos de interés. 


\section{Referencias}

1. Silva H. DSM-5: Nueva clasificación de los trastornos mentales. Rev Chil Neuro Psiquiat. 2014;52(supl 1):7-8

2. Lock J, La Via MC; American Academy of Child and Adolescent Psychiatry (AACAP) Committee on Quality Issues (CQI). Practice parameter for the assessment and treatment of children and adolescents with eating disorders. J Am Acad Child Adolesc Psychiatry. 2015;54(5):412-425.

3. Krogulska A, Nowicka D, Nowicki Z, Parzęcka M, Sakson-Słomińska A Kuczyńska R. A loss of consciousness in a teenage girl with anorexia nervosa due to polydipsia: case report and a minireview. Eat Weight Disord. 2019;24(5):969-974

4. Campbell K, Peebles R. Eating disorders in children and adolescents: state of the art review. Pediatrics. 2014;134(3):582-592.

5. Sieke EH, Rome ES. Eating disorders in children and adolescents: what does the gynecologist need to know? Curr Opin Obstet Gynecol. 2016;28(5):381-392.

6. Misra M, Klibanski A. Anorexia Nervosa and Its Associated Endocrinopathy in Young People. Horm Res Paediatr. 2016;85(3):147-157.

7. Boisseau CL. Identification and management of eating disorders in gynecology: menstrual health as an underutilized screening tool. Am J Obstet Gynecol. 2016;215(5):572-578.

8. Nigro N, Grossmann M, Chiang C, Inder WJ. Polyuria-polydipsia syndrome: a diagnostic challenge. Intern Med J. 2018;48(3):244-253.

9. Trimpou P, Olsson DS, Ehn O, Ragnarsson O. Diagnostic value of the water deprivation test in the polyuria-polydipsia syndrome. Hormones (Athens). 2017;16(4):414-422.
10. Sailer C, Winzeler B, Christ-Crain M. Primary polydipsia in the medical and psychiatric patient: characteristics, complications and therapy. Swiss Med Wkly. 2017;147:w14514.

11. Metropulos D, Antoon JW. Primary Polydipsia in a Child. Clinical Pediatrics. 2015;54(14):1396-98.

12. Teixeira B. Perestrelo J. Psychogenic polydipsia and hyponatremia - A side effect of psychosis: a review with a case report. 2016;65(3):300-303.

13. Kara, Koray \& Congologlu, Mehmet \& Durukan, İbrahim \& Karaman, Dursun. (2016). Psychogenic Polydipsia in an Adolescent with Eating Disorder: A Case Report. Gulhane Medical Journal. 58. 1. 10.5455/gulhane.152162.

14. Støving RK. Mechanisms in endocrinology: Anorexia nervosa and endocrinology: a clinical update. Eur J Endocrinol. 2019;180(1):R9-R27.

15. Aguiar DT, Monteiro C, Coutinho P. Recurrent rhabdomyolysis secondary to hyponatremia in a patient with primary psychogenic polydipsia. Rev Bras Ter Intensiva. 2015;27(1):77-81

16. Cruzado Díaz L., Kruger Malpartida H., Borda Olivos F. Polidipsia psicógena complicada con hiponatremia y rabdomiólisis: reporte de un caso. Rev medica hered. 2012;17(3):183.

17. Bhatia MS, Goyal A, Saha R, Doval N. Psychogenic Polydipsia - Managemen Challenges. Shanghai Arch Psychiatry. 2017;29(3):180-183. 


\section{SÍNDROME DE QUILOMICRONEMIA FAMILIAR (FCS): ${ }^{a}$}

Hereditario. Un cuadro clínico doloroso, recurrente y potencialmente fatal. A menudo subdiagnosticado.

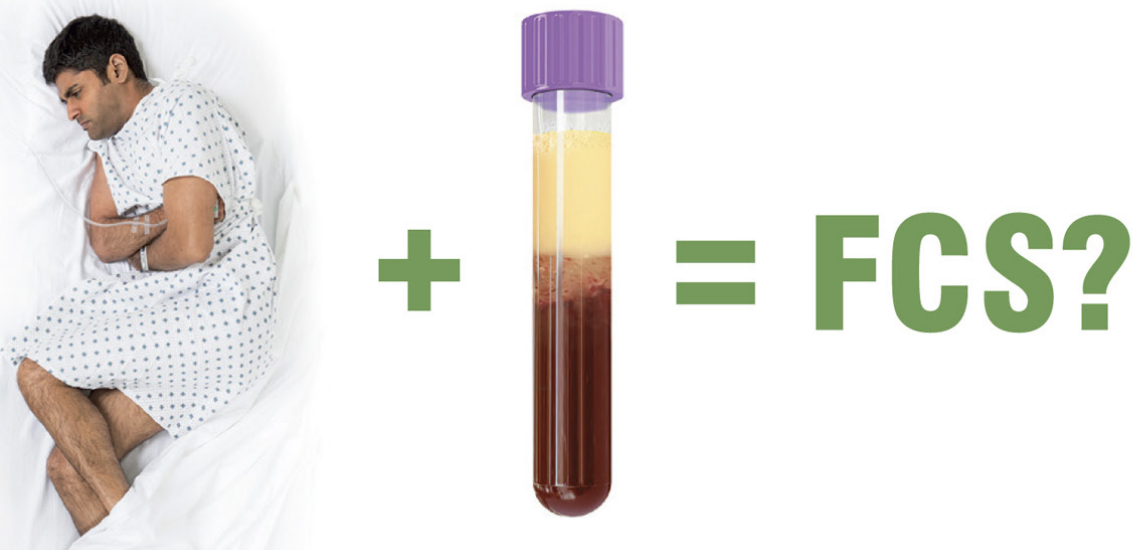

\section{Qué es FCS?}

- El FCS es una enfermedad genética de disfunción enzimática que inhibe el metabolismo de los quilomicrones. ${ }^{2,4}$

- La acumulación consiguiente de quilomicrones conduce a hipertrigliceridemia severa y las complicaciones asociadas. $^{5}$

- El FCS es una condición que expone a los pacientes al riesgo de pancreatitis aguda, cuadro potencialmente fatal, así como a múltiples morbilidades a largo plazo, tales como dolor abdominal crónico y diabetes pancreatogénica. ${ }^{1,2,5,6}$

\section{EL FCS PUEDE SER DIAGNOSTICADO CLÍNICAMENTE. BUSCAR: ${ }^{b}$}

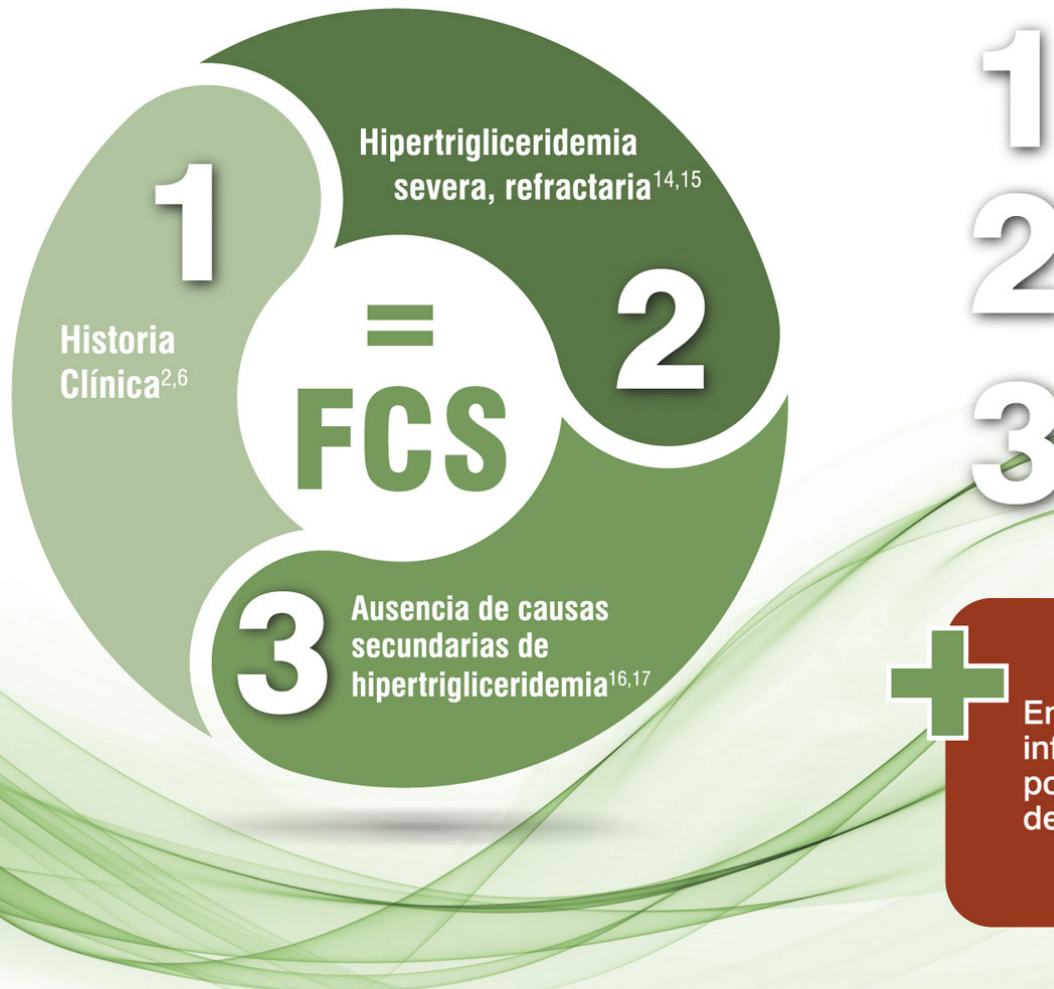

- Pancreatitis aguda y / o dolor abdominal (puede ser recurrente o crónico), sin otras causas explicables

- Triglicéridos en ayunas $>880 \mathrm{mg} / \mathrm{dL}$

- Sin respuesta, respuesta mínima a las terapias estándar.

- Exceso de consumo de alcohol diabetes no controlada, ingesta de ciertos medicamentos $\mathrm{u}$ otras condiciones médicas.

\section{EL ESTUDIO GENÉTICO ES ESENCIAL}

En caso tenga una sospecha o requiera mayor información. La toma de muestra será programada por el laboratorio Gencell Pharma en los laboratorios de referencia de su ciudad:

pacientes@labgencell.com

línea nacional 3183314900 / 3166904882

a: Otros nombres para el FCS incluyen deficiencia de lipoproteín lipasa, hiperlipoproteinemia tipo I e hiperlipidemia familiar. ${ }^{24,12,13}$ b. Criterio diagnóstico propuesto

Referencias:

1. Davidson et al. Expert Rev Cardiovasc Ther. 2017;15(5):415-423. 2. Brahm et al. Nat Rev Endocrinol. 2015;11:352-362. 3. Chait et al. Advances in Internal Medicine. 1992:249-273. 7. 2015:8(1):65-73. 11. Jacqueline htps'//globalgenes org/raredaily/my-jurney-to-fi nd-hope-with-familial-chylomicronemia-syndrome. Accessed June 9, 2017. 12. Tremblay et al Front. 2014:5:90. 13. Gotoda et al. J Atheroscler Thromb. 2012:19(1):1-12. 14. Tremblay et al. J Clin Lipidol. 2011:5(1):37-44. 15. Reiner et al. Eur Heart J. 2011:32(14):1769-1818. 16. Miller et al. Circulation. 2011;123(20):2292-2333. 17. Berglund et al. J Clin Endocrinol Metab. 2012;97(9):2969-2989. 18. Nawaz et al. Am J Gastroenterol. 2015;110(10):1497-1503 